\title{
Role of Neuromuscular Electrical Stimulation in Bell's Palsy: A Case Study of Three Patients
}

\author{
Nasrin Bharti ${ }^{1}$ \\ ${ }^{1}$ Centre for Physiotherapy and Rehabilitation Sciences, Jamia Millia Islamia, New Delhi - 110025, India
}

\begin{abstract}
Bell's palsy is an idiopathic, unilateral facial paralysis, caused by a malfunction anywhere along the facial nerve's peripheral portion, from the pons distally. Bell's palsy is treated by removing the cause of nerve injury, strengthening the face muscles, and restoring facial function. Physical therapy in the form of neuromuscular electrical stimulation (NMES), massage and facial exercises is used as adjuvant to hasten recovery. The aim of this study is to access of role of neuromuscular electrical stimulation (NMES) treatment in Bell's palsy patients. A detailed neurological assessment of three patients was done with emphasis on facial muscles and severity of paralysis was graded according to House Brackmann scale (HBS). Conventional physiotherapy was given in the form of electrical stimulation, facial massage, exercises and functional re-education on a daily basis. Patients were assessed at weekly and 1 months after the treatment. They experienced complete recovery within 1month follow-up, no recurrence was observed and all patients have normal facial movement. Physiotherapy in the form of NMES and facial exercises has a effective role in the early management of Bell's palsy.
\end{abstract}

Keywords: Bell's palsy; neuromuscular electrical stimulation; House Brackmann scale; physiotherapy

\section{INTRODUCTION}

Bell's palsy is an idiopathic, unilateral facial paralysis that affects about 23 people per 100,000 each year [1]. It's a term for a sudden onset, idiopathic facial paralysis caused by a malfunction anywhere along the facial nerve's peripheral portion, from the pons distally [2]. Bell's palsy, which affects both men and women equally, is the most prevalent cause of unilateral facial paralysis. Although the average age of onset is 40, it can happen at any age. Bell's palsy affects just a small percentage of children under the age of two [3]. Bell's palsy causes the palpebral fissure to expand, the nasolabial fold to flatten, and one corner of the mouth to droop when smiling. These signs occur on the same side of the face as the lesion. Bell's phenomenon is a classic sign in which the eyeball cannot close without moving upward and outward at the same time [4]. The cause of paralysis, is due to vasospasm after exposure to cold or draughts, vascular insufficiency and secondary ischemia or some form of viral infection. The risk of Bell's palsy is increased in patients with hypertension or Diabetics and in pregnant women (late in the pregnancy or in the early postpartum period) [5].

The main goals of treatment are to hasten healing, improve recovery, prevent corneal problems and other complications, and stop virus replication. Psychological assistance is also necessary. Patients must be followed up on a frequent basis. Patients with Bell's palsy should be referred to a specialist, and treatment should begin as soon as possible [6]. Bell's palsy is treated by removing the cause of nerve injury, strengthening the face muscles, and restoring facial function. Bell's palsy does not have a well-defined treatment or cure. Some cases are minor and may not require treatment because the symptoms normally go away in two weeks, whether or not 
medication is given. Antiviral drugs like acyclovir and anti-inflammatory drugs like the steroid like prednisolone are commonly used in medical treatment to combat viral infections and reduce nerve swelling.

Selective motor training is being used to facilitate symmetrical movement and control unwanted gross motor activity in neuromuscular retraining (synkinesis). Specific mirror exercises will provide sensory feedback to promote learning. When each muscle group is evaluated, the patient is instructed to examine the action of these muscles in the mirror and to make small symmetrical precise movements on the sound side to determine the correct response. As soon as the patient has identified the exact area of dysfunction, he or she can begin to exercise to enhance facial motions that are directed by the affected side, preserving isolated muscle response and improving coordination. Synkinesis is treated by inhibiting undesirable motions that occur during volitional and spontaneous movements, and by slowly moving the face without activating the aberrant movement [7].

The usefulness of physical therapies for Bell's palsy is debatable. Acupuncture, exercise, and electrical-stimulation are all common physical treatments. The data, however, is mixed when it comes to proving the efficacy of these therapies [8]. Hence the purpose of this study is to describe the role of treatment of neuromuscular electrical stimulation (NMES) in Bell's palsy which is commonly given in almost all the physical therapy settings as a part of conventional therapy.

\section{METHODOLOGY}

Patients diagnosed with Bell's palsy were referred to the outpatient physiotherapy department of G.B. Pant Hospital, New Delhi, India. Physiotherapy treatment was given to 3- patients, 72 hours after the onset of facial paralysis. A detailed neurological assessment was done with emphasis on facial muscles and severity of paralysis was graded according to House Brackmann scale (HBS). Conventional physiotherapy was given in the form of electrical stimulation, facial massage, exercises and functional re-education on a daily basis. Home program was also taught to the patients to be done 15 times each three times in a day. They were asked to close and open their eyes as tightly as possible, ballooning of the cheeks, chewing of chewing gum, straw sucking, showing of the teeth, pursing of the lips as in whistling and facial exercises simulating various facial expressions. These patients were assessed on regular basis after a certain period of time to look for the effects of treatment given to them.

\section{Grading}

To clinically assess the severity of peripheral facial nerve palsy various scoring systems are available. HBS grading systems rely on the evaluation of resting symmetry, degree of voluntary excursion of the facial muscles, and the degree of synkinesis. Bell's palsy was reported to progress to complete recovery (HBS $=1$ ), good recovery $(\mathrm{HBS}=2)$, and to partial recovery $(\mathrm{HBS}=3)$.

Table1: House-Brackmann facial nerve grading system (HBS)

\begin{tabular}{|l|l|l|l|l|}
\hline Grade & Degree & Description & At rest motion \\
\hline 1. & Normal & Gross formal facial function in all nerve & $\begin{array}{l}\text { Normal facial function } \\
\text { in all nerve branches }\end{array}$ & $\begin{array}{l}\text { Normal facial function in all nerve } \\
\text { branches }\end{array}$ \\
\hline 2. & Slight & $\begin{array}{l}\text { Slight weakness on close inspection, slight } \\
\text { asymmetry }\end{array}$ & $\begin{array}{l}\text { Normal tone and } \\
\text { asymmetry }\end{array}$ & $\begin{array}{l}\text { Forehead: slight to moderate movement } \\
\text { Eye: complete closure } \\
\text { with minimum effort } \\
\text { Mouth: slight } \\
\text { asymmetry }\end{array}$ \\
\hline 3. & Moderate & $\begin{array}{l}\text { Obvious but not disfiguring facial } \\
\text { asymmetry } \\
\text { Synkinesis is noticeable but not severe } \\
\text { may have hemi-facial spasm or } \\
\text { contracture }\end{array}$ & $\begin{array}{l}\text { Forehead: slight to moderate movement } \\
\text { Eye: complete closure } \\
\text { with effort } \\
\text { Mouth: slight } \\
\text { Weakness with maximum effort }\end{array}$ \\
\hline
\end{tabular}




\begin{tabular}{|l|l|l|l|l|}
\hline \multicolumn{9}{|c|}{ Table 1 Continued... } \\
\hline 4. & $\begin{array}{l}\text { Moderate } \\
\text { severe } \\
\text { symmetry }\end{array}$ & $\begin{array}{l}\text { Asymmetry is disfiguring \&/or obvious \& } \\
\text { facial weakness }\end{array}$ & $\begin{array}{l}\text { Forehead: no movement } \\
\text { Eye: incomplete closure } \\
\text { Mouth: asymmetrical with maximum } \\
\text { effort }\end{array}$ \\
\hline 5. & Severe & Only slight, barely noticeable movement & $\begin{array}{l}\text { Asymmetrical facial } \\
\text { appearance }\end{array}$ & $\begin{array}{l}\text { Forehead: no movement } \\
\text { Eye: incomplete closure } \\
\text { Mouth: slight movement }\end{array}$ \\
\hline 6. & $\begin{array}{l}\text { Total } \\
\text { Paralysis }\end{array}$ & No facial function & No facial function & No facial function \\
\hline
\end{tabular}

\section{CASE REPORTS}

\section{Case Study 1}

Demographic data

Name - XXX

Age - 20 yrs.

Gender - female

Occupation - student

Address - 16/4 kamla apartment, Moti Bagh Chief complaint - - facial weakness and asymmetry on the left side of the face.

History

1. Present history. - She found her face was distorted and deviated towards the right side, when she woke up in the next morning.

2. Receiving traditional treatment- no

3. Past medical history - no relevant history was present

4. Socioeconomic status - middle class

5. Psychological impact - depressed

6. Medication - antiviral drug (acyclovir, prednisolone for 6 days)

7. Observations/associated conditions facial asymmetry and bell's phenomenon are present on the left side of the face.

8. Pain examination:

Location - pain behind the ear was present before the onset of paralysis.

VAS - 2

Intensity - mild

9. Sensation

Light touch - normal

Temperature - normal

Any abnormal sensory feelings - no

10. Neurological examination (examination of cranial nerve 7)

Test motor function of facial muscles raise eyebrows, frown, show teeth, smile, close eye tightly, puff out both cheeks - inability to close complete eye, forehead no movement, drooping corner of mouth, difficulty with speech articulation.

Test to anterior $2 / 3$ rd of the tongue normal

11. House-Brackmann Facial Grading System - Score of 4

12. Functional Status - difficulty in mastication of food and talking

13. Diagnosis - Bell's palsy on the left side of the face.

\section{Case Study 2}

Demographic data

Name - XXX

Age - 11 yrs

Gender - male

Occupation - student

Address - vikash apartment, Lajpat nagar

Chief complaint - not being able to close his left eye and deviation of the mouth to the right side of the face. He first noted the sign day after the onset of paralysis.

History

1. Present history- he noted facial asymmetry and also complained of facial deviation was present in the next morning.

Receiving traditional treatment - no

2. Past medical history- history of eye infection or trauma was present

3. Family history- no relevant history

4. Socioeconomic status-

5. Psychological impact- no

6. medication- antiviral medicine (acyclovir, prednisolone for 6 days)

7. Observation /associated conditions: Left muscles of facial expression didn't participate when the child smiled.

No swelling present

Left eye close partially

Eyelid lags with flattening of the nasal labial fold on the left side 
8. Pain examination

VAS - experience no pain before the onset of paralysis

Location- pain in the mastoid region Intensity- mild

9. Sensation

Light touch- normal

Temperature- normal

10. Neurological examination (cranial nerve 7)

Inability to wrinkle the forehead, left eye close partially, drooping corner of the mouth

11. House-Brackmann Facial Grading system- score of 3.

12. diagnosis- Bell's palsy on the left side of the face

\section{Case Study 3}

Demographic data

Name - XXX

Age - 19 yrs

Gender - male

Occupation - student

Address - GNIT boys hostel, Greater Noida Chief complaint - facial symmetry is normal but facial muscle weakness is present.

History

1. Present history- he felt weakness over the left side of the face, due to which he had difficulty in moving left corner of the mouth, not being able to close his left eye completely and unable to smile since the next day onset of paralysis .

Receiving traditional treatment - no

2. Past medical history- no relevant history

3. Family history- no relevant history

4. Socioeconomic status-

5. Psychological impact- no

6. Medication- antiviral and antiinflammatory drugs (acyclovir, prednisolone for 6 days)

7. Observation /associated conditions: Difficulty is found to move the eyelid, move the corner of the mouth and nose, loss of wrinkles of the forehead on the left side of the face.
Left eye close partially, Eyelid lags with flattening of the nasal labial fold on the left side.

No swelling present

8. Pain examination

VAS- no pain was present

Location-

Intensity-

9. Sensation

Light touch- normal

Temperature- normal

10. Neurological examination (cranial nerve 7)

Inability to wrinkle the forehead, left eye close partially, drooping corner of the mouth

11. House-Brackmann Facial Grading system- score of 3.

12. diagnosis- Bell's palsy on the left side of the face

\section{PHYSIOTHERAPY MANAGEMENT}

The application of electrical current to motor nerves to cause contractions in the muscles they innervate is known as neuromuscular electrical stimulation (NMES). NMES is commonly used in physical medicine and rehabilitation facilities to boost muscle strength development, prevent or decrease muscle atrophy and fibrosis, and improve muscle re-education, including sensory awareness and volitional muscle control [9]. The electrical stimulation causes action potentials to be generated in the motor neurons, which then propagate along the motor neurons to the muscle. The action potentials cause the muscle to contract when they reach it. The current intensity and length of the impulses are sufficient to begin contraction of the denervated muscle. The slowly growing current might cause denervated muscle to contract. The shortest impulse utilized to treat denervated muscle is one that lasts 100 milliseconds (ms). Interrupted galvanic (D.C) current (continuous unidirectional flow of charged particles) with a long duration $>10 \mathrm{~ms}$ and a frequency of 30 per mint are utilized to stimulate denervated muscles [10]. 
Electrode Placement: In the supine lying posture, an inactive electrode was inserted at the nape of the neck, and the face muscles were stimulated with a pen electrode (active electrode). Current intensity is gradually increased until a good muscle contraction is achieved at the maximum point of each surge, which is then repeated to induce alternate muscle contraction and relaxation.

Impulse: Rectangular impulses with a duration of 100 milliseconds are the shortest and require a frequency of roughly 30 milliseconds. They feature a sharp increase and fall in strength. It has the advantage of obtaining a contraction of a denervated muscle with less sensory stimulus, hence eliminating undesired contractures of innervated muscles. A slowly rising current can cause denervated muscle to contract, which is a physiological impact of intermittent D.C current. The shocks are repeatedly delivered in order to induce a brick muscle twitch followed by quick relaxation. Electrical stimulation produces the maximum contraction with the least amount of electricity at motor sites.

\section{Motor points of facial nerve;}

- Temporal branch -frontalis, corrugators, orbicularis oculi.

- Zygomatic branch- orbicularis oculi, nasalis

- Buccal branch- procerus, risorius, buccinators, levator labii superioris, levator anguli oris, nasalis, orbicularis oris.

- Marginal Mandibular branch- mentalis, depressor labii inferioris, depressor anguli oris.

- Cervical branch

Massage therapy: improves circulation and may prevent contracture and improves facial asymmetry. Commonly massage techniques include - stroking, effleurage, finger or thumb kneading and hacking.

Eyes Protection: artificial tears, ophthalmic ointment before sleep and eyeglasses are usually used to protect from light, dust and wind.

Neuromuscular re-education: based on an understanding of the physiological pattern of muscle actions. Exercises which help to integrate involved-side muscle activity with the uninvolved side provide opportunities for the involved-side muscle to exercise, particularly in desired patterns of movement, and promote coordination. The exercise program usually consists of 5 to 10 repetitions of 3 to 5 exercises to be done twice daily.

\section{DISCUSSION}

The clinical aspects and physiotherapy care for the treatment of Bell's palsy patients are described in these case reports. These studies found that after treatment with NMES, facial massage, and facial movement exercises (with visual feedback from a mirror) to improve asymmetry and synkinesis as suggested by clinical practice and scientific literature, the characteristics of people with Bell's Palsy improved in their initial symptoms. Patients with facial paralysis have historically received physical therapy in the form of basic facial exercises or electrical stimulation. However, there are just a few randomized controlled trials on the efficacy of physical therapy [11].

When it comes to the effects of ES on motor neuron regeneration, the evidence is mixed. According to a research by Targan et al. ES may aid partial reinnervation in patients with persistent facial paresis [12]. Farragher et al., employed ES on 40 patients with a pulse duration of $80 \mathrm{~ms}$ and a frequency and pattern that matched healthy facial muscles and find that ES could help with facial palsy [13]. The conservative approach to facial rehabilitation is facial neuromuscular re-education. Improvements in deficits related with facial paralysis have been demonstrated as a result of face neuromuscular re-education [14]. An evaluation of face impairments and functional restrictions, guided training 
sessions of correct movement patterns, and instruction in a specialized facial movement exercise program are all part of facial neuromuscular re-education [15].

For individuals with face neuromotor abnormalities, however, neuromuscular re-education shown to be better or equivalent to the comparison standard of care. Patients with facial paralysis have traditionally received physical therapy in the form of basic facial exercises or ES [16]. Traditional facial paralysis treatment differs from face neuromuscular re-education procedures. As a result, the exercise regimen evolves over time as the patient's deficits improve. Exercises that encourage mass contraction of muscles connected to more than one facial emotion are excluded from the facial neuromuscular re-education exercise program, which emphasizes accuracy of facial movement patterns and isolated muscle control. The amount of exercise repetitions and the frequency of the exercise program in our method are determined by treatment-based categories based on the patient's disability.

ES has been suggested to interfere with neural regeneration following peripheral nerve injury, and there are no studies showing its efficacy with facial muscles in the literature. Furthermore, patients who are exposed to ES for a short period of time may show more synkinesis and mass action than those who are not. Due to their tiny size and close proximity, it is difficult to elicit an independent contraction of the face muscles by ES.

\section{CONCLUSION}

This study was done to find out the role of NMES in Bell's palsy. Patients with Bell's palsy continue to be treated with traditional therapy in the form of NMES, massage and exercises. However recent research and literature suggests that advanced approaches like neuromuscular retraining (NMR/PNF) techniques have shown to be more effective compared to the conventional physiotherapy. Hence, we should incorporate these re-education approaches in Bell's palsy rather than the traditional stimulation, massage and exercises.

\section{ACKNOWLEDGEMENT}

I would like to thank Dr. Nisar Ahmad and Professor Ejaz Hussain, Centre for Physiotherapy \& Rehabilitation Sciences Jamia Millia Islamia, for providing all the facilities. The author is also thankful to the Physiotherapy Department of G.B. Pant Hospital, New Delhi, India.

\section{Conflict of Interest: None}

\section{Source of Funding: None}

\section{REFERENCES}

1. Marson AG, Salinas,R. Bell's palsy. The Western journal of medicine. 2000; 173(4): 266-268.

2. Olsen KD. Facial nerve paralysis 1. General evaluation, Bell's palsy. Postgrad Med. 1984; 75: 219-225.

3. Ciorba A, Corazzi V, Conz V, Bianchini C, Aimoni C. Facial nerve paralysis in children. World journal of clinical cases. 2015; 3(12): 973-979.

4. Somasundara D, Sullivan F. Management of Bell's palsy. Australian prescriber. 2017; 40(3): 94-97.

5. Ahmed A. When is facial paralysis Bell palsy? Current diagnosis and treatment. Cleve Clin J Med. 2005; 72: 398-401.

6. Holland NJ, Weiner GM. Recent developments in Bell's palsy. BMJ (Clinical research ed.). 2004; 329(7465): 553-557.

7. Diels HJ. Facial paralysis: is there a role for a therapist? Facial Plast Surg. 2000; 16(4): 361-364.

8. Haltiwanger E, Huber $\mathrm{T}$, Chang JC, Gonzalez-Stuart A. Case study of Bell's palsy applying complementary treatment within an occupational therapy model. Occupational therapy international, 2009; 16(1): 71-81.

9. Carnaby-Mann GD, Crary MA. Examining the evidence on neuromuscular electrical stimulation for swallowing: a metaanalysis. Archives of otolaryngology--head \& neck surgery. 2007; 133(6): 564-571. 
10. Limbu TK, Nepal G. Current practice patterns in the management of bell's palsy amongst physiotherapists in Nepal: A crosssectional study. Annals of Physical and Rehabilitation Medicine. 2018; e519.

11. Cardoso JR, Teixeira EC, Moreira MD, Fávero FM, Fontes SV, Bulle de Oliveira AS. Effects of exercises on Bell's palsy: systematic review of randomized controlled trials. Otology \& neurotology: official publication of the American Otological Society, American Neurotology Society [and] European Academy of Otology and Neurotology, 2008; 29(4): 557-560.

12. Targan R, Alon G, Kay SL. Effect of longterm electrical stimulation on motor recovery and improvement of clinical residuals in patients with unresolved facial nerve palsy. Otolaryngol Head Neck Surg. 2000; 122, 246-252.

13. Farragher D, Kidd GL, Tallis R. (1987). Eutrophic electrical stimulation for Bell's palsy. Clinical Rehabilitation. 1987; 1(4): 265-271.

14. Ross BG, Fradet G, Nedzelski J M. Development of a sensitive clinical facial grading system. Otolaryngol Head Neck Surgery. 1996; 114, 380-386.

15. Brach JS, VanSwearingen JM. Physical therapy for facial paralysis: a tailored treatment approach. Physical therapy. 1999; 79(4): 397-404.

16. Teixeira LJ, Valbuza JS, Prado GF. Physical therapy for Bell's palsy (idiopathic facial paralysis). Cochrane Database Syst Rev. 2011; 12: CD006283.

How to cite this article: Bharti N. Role of neuromuscular electrical stimulation in bell's palsy: a case study of three patients. International Journal of Science \& Healthcare Research. 2021; 6(4): 235-241. DOI: https:// doi.org/10.52403/ijshr.20211034 